\title{
DOES ATTACHMENT TO GOD PREDICT MINDFULNESS?
}

\author{
Tracy A. Freeze ${ }^{1}$, Lisa Best ${ }^{2}$, Cecile Proctor ${ }^{3}$, \& Enrico DiTommaso ${ }^{2}$ \\ ${ }^{1}$ Ph.D., Department of Psychology, Crandall University (Canada)* \\ ${ }^{2}$ Ph.D., Department of Psychology, University of New Brunswick Saint John (Canada) \\ ${ }^{3}$ M.A., Department of Psychology, University of New Brunswick Saint John (Canada)
}

\begin{abstract}
In the past decade, a plethora of research has focused on examining the relationship between mindfulness and a variety of psychological constructs. In general, research has indicated that mindfulness is positively associated with health and well-being (see Nagy \& Baer, 2017). Simply defined, mindfulness is attentiveness in the present moment (Baer, 2003). Further, Linehan (1993) noted that the object and the quality of attentiveness are essential components of mindfulness. Attachment to God is a construct that also has an "object" of the individual's attention (i.e., God) and individual differences in the "quality" of that attention (i.e., varying levels of attachment security). Given that individual differences in attachment to God have also been examined in relation to psychological constructs and were predictive of well-being (e.g., Freeze \& DiTommaso, 2014, 2015), an association between attachment to God and mindfulness may be hypothesized. However, few, if any, studies have examined mindfulness in relation to either religious belief or attachment to God. The aim of the present study was to determine whether individual differences in attachment to God predict five facets of mindfulness (i.e., observing, describing, acting with awareness, non-judgmental inner experience, and non-reactivity). It was hypothesized that insecure attachment to God would be negatively associated with mindfulness. Five hundred and sixty-nine undergraduate students at a secular university completed questionnaire packages that included demographics, questions about their religious belief, the Social Readjustment Rating Scale, the Attachment to God Scale, and the Five Facet Mindfulness Scale. Five Bonferroni-corrected hierarchical multiple regression analyses were conducted. After controlling for stress and belief in God, higher levels of anxious and avoidant attachment to God predicted lower levels of the describing facet of mindfulness. Higher levels of anxious attachment to God predicted lower levels of both acting with awareness and non-judgmental inner experience. Neither anxious attachment to God nor avoidant attachment to God significantly predicted the observing and non-reactivity facets of mindfulness. Implications of these findings and directions for future research will be discussed.
\end{abstract}

Keywords: Mindfulness, attachment to God, religion.

\section{Introduction}

In the past few decades, researchers have separately examined both mindfulness and attachment to God as predictors of well-being (e.g., Nagy \& Baer, 2017; Freeze \& DiTommaso, 2014, 2015). However, few, if any, have examined the relationship between attachment to God and mindfulness, despite the fact that both share "attentiveness" as a component (Linehan, 1993; Granqvist, Mikulincer, \& Shaver, 2010). Linehan (1993) suggested that the object and the quality of attentiveness are essential components of mindfulness. Attachment theory also examined the object and quality of an individual's attentiveness, with God as the "object" and individual differences in the security of that relationship as the "quality" (Granqvist et al., 2010). The purpose of the current study was to examine the relationship between individual differences in the attachment to God relationship and the various facets of mindfulness.

Attachment to God is an extension of attachment theory that examines the close emotional bond that some human beings have with God (Kirpatrick \& Shaver, 1990). Following the two dimensions of adult human attachment, anxious attachment to God is a form of an insecure relationship in which God's responsivity to human need is experienced as inconsistent, and there is anxiety about abandonment (Granqvist et al, 2010). Similarly, avoidant attachment to God is also a type of insecure relationship but, in this case, God is seen as distant and uncaring, and intimacy with God is avoided (Granqvist et al., 2010). As with attachment theory in general, research on attachment to God has indicated clear links between the

${ }^{*}$ Dr. Tracy Freeze is now at Horizon Health Network, New Brunswick, Canada. 
quality of the relationship and psychological outcomes. For instance, insecure attachment to God in people of faith has predicted psychological outcomes. For instance, insecure attachment to God in people of faith has predicted emotional distress (Freeze \& DiTommaso, 2014, 2015), and secure attachment to God has predicted psychological health (Leman, Hunter, Fergus, \& Rowatt, 2018).

Recently, research on mindfulness has also indicated links between facets of mindfulness and psychological health (e.g., Nagy \& Baer, 2017; Marzabadi, Mills, \& Valikhani, 2018). Baer (2003) defined mindfulness as attentiveness in the present moment and subsequently developed the Five Facet Mindfulness Questionnaire (Baer, Smith, Hopkins, Krietemeyer, \& Toney, 2006). The five facets identified were: Observing (being aware of internal/external stimuli; e.g., bodily sensations, sounds, smells), Describing (the ability to put one's experience into words and describe the emotions experienced), Acting with Awareness (awareness of actions without the mind wandering), Non-Judgement of Inner Experience (allowing internal thoughts/feelings without negative/positive evaluations of the thoughts/feelings), and Non-Reactivity to Inner Experience (not reacting to internal thoughts/feelings).

In both mindfulness and attachment to God, the inadequate "object" and "quality" of attentiveness predicts lower levels of well-being. Therefore, the purpose of the current study was to examine the associations between attachment to God and mindfulness facets. We hypothesized that, after controlling for stress and belief in God, higher levels of insecure attachment to God would be associated with lower levels of the five facets of mindfulness.

\section{Method}

\subsection{Measures}

Participants completed a questionnaire package that included demographic questions as well as a question about their current belief in God. The questionnaire package also included the Social Readjustment Rating Scale (SRRS; Holmes \& Rahe, 1967), the Attachment to God Inventory (AGI; Beck \& McDonald, 2004), and the Five Facet Mindfulness Questionnaire (FFMQ; Baer et al., 2006). The AGI is a 28-item scale that measures the domains of anxious attachment to God (AGX) and avoidant attachment to God (AGV). The FFMQ is a 39-item questionnaire that measures five facets of mindfulness (observe, describe, act with awareness, nonjudge, and nonreact). Cronbach's alpha for all of the measures ranged from .76 to .91 .

\subsection{Participants}

Five hundred and sixty-nine undergraduate students were recruited via the SONA recruitment system. Students received one bonus point for completing the study. Participants ranged in age from 18-58 with a mean age of $20.98(S D=5.65)$. The participants were primarily white or Caucasian $(84.7 \%)$ and female (72.1\%). Participants identified their belief about God as: "I know that God really exists, and I have no doubt about it" (Belief 1; 22.1\%); "While I have doubts, I feel that I do believe in God" (Belief 2; $24.5 \%$ ); "I find myself believing in God some of the time, but not at other times" (Doubt - reference group; $17.3 \%$ ); "I don't know if there is a God, and I don't believe there is any way to find out" (Agnostic; 20.2\%); "I do not believe in God" (Atheist; 14.6\%).

\section{Results}

Five Bonferroni-corrected hierarchical multiple regression analyses were conducted. The control variables were stress (SRRS) and belief about God. Belief about God was coded with the mid-point (reflecting the most doubt) as the reference group. The control variables significantly predicted the Observing facet of mindfulness $(F(5,551), p<.001)$. Higher levels of stress in the past year predicted higher levels of Observing $(t=4.40, p<.001)$. Stress, along with Belief 1also predicted Non-Judgemental Inner Experience $(F(5,551), p<.001)$. Higher levels of Belief 1 (participants who believe in God without doubt) compared to the reference group (Doubt) predicted higher levels of Non-Judgemental Inner Experience $(t=4.40, p=.002)$. Higher levels of stress predicted lower levels of Non-Judgemental Inner Experience $(t=-5.38, p<.001)$. Finally, the control variables significantly predicted acting with awareness $(F(5,551), p<.001)$. Higher levels of Belief 1 predicted higher levels of Acting with Awareness $(t=4.89$, $p<.001)$.

After controlling for stress and belief in God, higher levels of ANX $(t=-5.03, p<.001)$ and AGV $(t=-4.03, p<.001)$ predicted lower levels of the Describing facet of mindfulness $(F(2,549)=16.33$, $p<.001)$. Further, higher levels of AGX predicted lower levels of both Acting with Awareness $(F(2,549)$ $=21.40, p<.001) ; t=-6.54, p<.001)$ and Non-Judgemental Inner Experience $(F(2,549)=6.97, p=.001$; $t=-3.61, p<.001)$. Neither AGX nor AGV significantly predicted the Observing and Non-Reactivity facets of mindfulness. 
Table 1. Hierarchical Multiple Regression Analyses of Attachment to God Predicting Facets of Mindfulness.

\begin{tabular}{|c|c|c|c|c|c|c|c|c|c|c|}
\hline & \multicolumn{2}{|c|}{ Observe } & \multicolumn{2}{|c|}{ Describe } & \multicolumn{2}{|c|}{ Aware } & \multicolumn{2}{|c|}{ Nonjudge } & \multicolumn{2}{|c|}{ Nonreact } \\
\hline Step 1 & $\boldsymbol{\beta}$ & $s r^{2}$ & $\boldsymbol{\beta}$ & $s r^{2}$ & $\boldsymbol{\beta}$ & $s r^{2}$ & $\boldsymbol{\beta}$ & $s r^{2}$ & $\beta$ & $s r^{2}$ \\
\hline Stress & $.185^{* *}$ & .034 & -.011 & .000 & -.110 & .012 & $-.223^{* *}$ & .049 & .054 & .003 \\
\hline Belief1 & -.040 & .001 & .148 & .012 & $.270^{* *}$ & .041 & $.174^{*}$ & .017 & .115 & .007 \\
\hline Belief2 & .005 & .000 & .048 & .001 & .108 & .006 & .080 & .003 & .082 & .004 \\
\hline Agnostic & .049 & .001 & .006 & .000 & .089 & .004 & .070 & .003 & .001 & .000 \\
\hline Atheist & -.031 & .001 & .110 & .008 & .028 & .000 & .042 & .001 & -.037 & .001 \\
\hline$R^{2}$ & \multicolumn{2}{|c|}{$.040^{* *}$} & \multicolumn{2}{|c|}{.021} & \multicolumn{2}{|c|}{$.058^{* *}$} & \multicolumn{2}{|c|}{$.063^{* *}$} & \multicolumn{2}{|c|}{.023} \\
\hline Step 2 & & & & & & & & & & \\
\hline AGX & .006 & .000 & $-.237^{* *}$ & .042 & $-.299^{* *}$ & .068 & $-.169^{* *}$ & .022 & -.052 & .002 \\
\hline AGV & -.050 & .001 & $-.227^{* *}$ & .027 & -.090 & .004 & -.007 & .000 & -.021 & .000 \\
\hline$R^{2} \Delta$ & \multicolumn{2}{|c|}{.002} & \multicolumn{2}{|c|}{$.055^{* *}$} & \multicolumn{2}{|c|}{$.068^{* *}$} & \multicolumn{2}{|c|}{$.023^{* *}$} & \multicolumn{2}{|c|}{.002} \\
\hline
\end{tabular}

\section{Conclusions}

After controlling for stress and belief in God, insecure attachment to God predicted the Describing, Acting with Awareness, and Non-Judgmental facets of mindfulness. These findings support theoretical work suggesting that insecure attachment has a deleterious effect on emotion regulation (Bowlby, 1980). Further, it supports empirical work (Pickard, Caputi, \& Grenyer, 2016) indicating that mindfulness-based interventions may lead to better psychological outcomes for individuals with insecure attachment relationships. Future research should focus on examining mindfulness-based interventions and their potential ability to attenuate the impact of insecure attachment to God.

\section{References}

Baer, R. A. (2003). Mindfulness training as a clinical intervention: A conceptual and empirical review. Clinical Psychology: Science and Practice, 10(2), 125-143.

Baer, R. A., Smith, G. T., Hopkins, J., Krietemeyer, J., \& Toney, L. (2006). Using self-report assessment methods to explore facets of mindfulness. Assessment, 13, 27-45.

Beck, R., \& McDonald, A. (2004). Attachment to God: The Attachment to God Inventory, tests of working model correspondence, and an exploration of faith group differences. Journal of Psychology and Theology, 32(2), 92-103.

Bowlby, J. (1980) Attachment and Loss, Vol. 3, Loss. London. Hogarth Press.

Freeze, T. A., \& DiTommaso, E. (2014). An examination of attachment, religiousness, spirituality and well-being in a Baptist faith sample. Mental Health, Religion \& Culture, 17(7), 690-702.

Freeze, T. A., \& DiTommaso, E. (2015). Attachment to God and church family: Predictors of spiritual and psychological well-being. Journal of Psychology \& Christianity, 24(1), 60-72.

Granqvist, P., Mikulincer, M., \& Shaver, P. R. (2010). Religion as attachment: Normative processes and individual differences. Personality and Social Psychology Review, 14(1), 49-59.

Holmes, T. H., \& Rahe, R. H. (1967). The social readjustment rating scale. Journal of Psychosmatic Research, 11, 213-218.

Kirkpatrick, L. A., \& Shaver, P. R. (1990). Attachment theory and religion: Childhood attachments, religious beliefs, and conversion. Journal for the Scientific Study of Religion, 29(3), 315-334.

Leman, J., Hunter, W., Fergus, T., \& Rowatt. (2018). Secure attachment to God uniquely linked to psychological health in a national, random sample of American adults. The International Journal for the Psychology of Religion, 28(3), 162-173.

Marzabadi, E., Mills, P. J., \& Valikhani, A. (2018). Positive personality: Relationships among mindful and grateful personality traits with quality of life and health outcomes, Current Psychology. doi:10.1007/s12144-018-0080-8

Nagy, L. M., \& Baer, R.A. (2017). Mindfulness: What should teachers of psychology know?. Teaching of Psychology, 44(4), 353-359.

Pickard, J. A., Caputi, P., \& Grenyer, B. F. (2016). Mindfulness and emotional regulation as sequential mediators in the relationship between attachment security and depression. Personality and Individual Differences, 99, 179-183. 